\title{
Abstract: Phase-Sensitive Region-of-Interest Computed Tomography
}

\author{
Lina Felsner ${ }^{1}$, Martin Berger ${ }^{3}$, Sebastian Kaeppler ${ }^{1}$, Johannes Bopp ${ }^{1}$, \\ Veronika Ludwig ${ }^{2}$, Thomas Weber ${ }^{2}$, Georg Pelzer ${ }^{2}$, Thilo Michel ${ }^{2}$, Andreas \\ Maier $^{1}$, Gisela Anton ${ }^{2}$, Christian Riess ${ }^{1}$ \\ ${ }^{1}$ Pattern Recognition Lab, Computer Science, Univ. of Erlangen-Nürnberg \\ ${ }^{2}$ Erlangen Centre for Astroparticle Physics, Univ. of Erlangen-Nürnberg \\ ${ }^{3}$ Siemens Healthcare GmbH \\ lina.felsner@fau.de
}

X-ray Phase-Contrast Imaging (PCI) is a novel imaging technique that can be implemented with an grating interferometer. PCI is compatible with clinical $\mathrm{X}$-ray equipment, and yields in addition to an absorption image also a differential phase image and a dark-field image. Computed Tomography (CT) of the differential phase can in principle provide high-resolution soft-tissue contrast. Recently, grating-based PCI took several hurdles towards clinical implementation by addressing, for example, acquisition speed, high X-ray energies, and system vibrations. However, a critical impediment in all grating-based systems lies in limits that constrain the grating diameter to few centimeters. Such a small field of view is a major challenge, since the object is typically larger, which leads to truncation in the projection images and therefore artifacts in the reconstruction.

In our work, we propose a system and a reconstruction algorithm to correct for phase truncation artifacts, and to obtain quantitative phase values in a clinically compatible way [1]. We propose to perform a phase-sensitive region-ofinterest CT within a full-field absorption CT. An attenuating collimator can be used to mount the gratings, leading to less dose in the peripheral region outside of the gratings. Furthermore we propose an algorithm to correct for the phase value truncation by using the absorption information. Our method first performs a segmentation of the materials, which allows to obtain an estimate of their respective phase values. Then, a non-truncated sinogram is extrapolated from the truncated sinogram and the estimated phase values.

Our method is robust, and shows high-quality results on simulated data and on a biological mouse sample. The work is a proof of concept showing the potential to use PCI in CT on large specimen, such as humans, in clinical applications.

\section{References}

1. Felsner L, Berger M, Kaeppler S, et al. Phase-sensitive region-of-interest computed tomography. Proc MICCAI. 2018;1:37-144. 